\title{
Case report for an adolescent with germline RET mutation and alveolar rhabdomyosarcoma
}

\author{
Kenneth A. Crawford, ${ }^{1}$ Noah E. Berlow, ${ }^{1}$ Jennifer Tsay, ${ }^{2}$ Michael Lazich, ${ }^{2}$ \\ Maria Mancini, ${ }^{3}$ Christopher Noakes, ${ }^{3}$ Tannie Huang, ${ }^{4}$ and Charles Keller ${ }^{1}$ \\ ${ }^{1}$ Children's Cancer Therapy Development Institute, Beaverton, Oregon 97005, USA; ${ }^{2} 2016$ Pediatric Cancer \\ Nanocourse, Children's Cancer Therapy Development Institute, Beaverton, Oregon 97005, USA; ${ }^{3}$ Champions \\ Oncology, Hackensack, New Jersey 07601, USA; ${ }^{4}$ Kaiser Permanente Santa Clara Medical Center, Santa Clara, \\ California 95051, USA
}

\begin{abstract}
In this case report we evaluate the genetics of and scientific basis of therapeutic options for a 14-yr-old male patient diagnosed with metastatic PAX3-FOXO1 fusion positive alveolar rhabdomyosarcoma. A distinguishing genetic feature of this patient was a germline RET C634F mutation, which is a known driver of multiple endocrine neoplasia type 2A (MEN2A) cancer. Through sequential DNA and RNA sequencing analyses over the patient's clinical course, a set of gene mutations, amplifications, and overexpressed genes were identified and biological hypotheses generated to explore the biology of RET and coexisting signaling pathways in rhabdomyosarcoma. Somatic genetic abnormalities identified include CDK4 amplification and FGFR4 G388R polymorphism. Because of the initial lack of patient-derived primary cell cultures, these hypotheses were evaluated using several approaches including western blot analysis and pharmacological evaluation with molecularly similar alveolar rhabdomyosarcoma cell lines. Once a primary cell culture became available, the RET inhibitor cabozantinib was tested but showed no appreciable efficacy in vitro, affirming with the western blot negative for RET protein expression that RET germline mutation could be only incidental. In parallel, the patient was treated with cabozantinib without definitive clinical benefit. Parallel chemical screens identified PI3K and HSP9O as potential tumor-specific biological features. Inhibitors of PI3K and HSP9O were further validated in drug combination synergy experiments and shown to be synergistic in the patient-derived culture. We also evaluated the use of JAK/STAT pathway inhibitors in the context of rhabdomyosarcomas bearing the FGFR4 G388R coding variant. Although the patient succumbed to his disease, study of the patient's tumor has generated insights into the biology of RET and other targets in rhabdomyosarcoma.
\end{abstract}

2020 Crawford et al. This article is distributed under the terms of the Creative Commons Attribution-NonCommercial License, which permits reuse and redistribution, except for commercial purposes, provided that the original author and source are credited.

Ontology term: alveolar rhabdomyosarcoma

Published by Cold Spring Harbor Laboratory Press

doi:10.1101/mcs.a004853

[Supplemental material is available for this article.]

\section{INTRODUCTION}

Rhabdomyosarcoma (RMS) is the most common childhood soft-tissue sarcoma and often also occurs in adolescents and young adults under age 40. In the United States, alveolar RMS (ARMS) has an annual incidence of approximately one incident per million persons per year across all age groups ( 350/yr). The ARMS subtype accounts for $20 \%-30 \%$ of all RMS tumors and represents $\sim 1 \%$ of all pediatric cancers. ARMS is often described by the defining gene fusion event that initiates the ARMS: the more prevalent PAX3:FOXO1-positive 
variant and the less common PAX7:FOXO1-positive subtype. ARMS can also be driven by atypical fusions (PAX3:NCOA1, PAX3:INO8) or can be fusion-negative.

The standard treatment for RMS consists of surgery, radiation, and intensive chemotherapy (Hawkins et al. 2013). Specific therapeutic protocols have been developed by the Intergroup Rhabdomyosarcoma Study Group (IRSG) for patients based on their risk group with the goal of reducing therapy related toxicities for patients with low risk of recurrent disease. Patient risk group is determined by histological criteria (e.g., embryonal RMS vs. alveolar RMS, and local vs. metastatic disease) and molecular profiling (such as fusion status), as well as tumor site and extent of disease. The different risk groups have vastly different outcomes with low-risk patients having an overall survival rate of $>90 \%$ (Raney et al. 2011). On the other hand, ARMS patients in the high-risk, metastatic disease group have poor 5-yr event-free survival (EFS) rates, with EFS rates for fusion-negative ARMS at 29\%, ARMSunknown at $17 \%$, ARMS-PAX7 at 17\%, and ARMS-PAX3 at $8 \%$ (Rudzinski et al. 2017). A significant fraction (15\%) of patients with RMS present with metastatic disease denoted IRSG Stage IV (IRSG-IV) at the time of diagnosis (Oberlin et al. 2008). Current RMS research is often focused on PI3K/mTOR inhibitors as an effective therapeutic strategy, particularly in combination with additional agents such as chemotherapy (clinical trial NCT01222715). However, long-term benefits are still unclear, thus effective treatment strategies for patients with metastatic disease remains an area of clinical need.

This case presentation describes a 14-yr-old male with advanced metastatic ARMS. At first, high-throughput genomic sequencing of the patient's excised tumor was performed to generate hypotheses for drivers of tumor maintenance/progression. In the early phase of this analysis, patient-derived cell cultures were not available, so genetically selected surrogate experimental cell model systems were used to test hypotheses. Although the patient's disease was initially responsive to standard chemotherapy and radiation, over time the patient's disease progressed. Disease progression necessitated further surgeries, which provided additional tumor tissue for further genetic analysis, protein expression analysis, and cell model generation that enabled drug screening and drug synergy studies. In this article we report our posthumous results that, for this patient, genetic analysis alone may have resulted in false positives, whereas functional genomics (chemical drug screens plus genomics) may have been a more viable method for identifying effective therapeutic interventions.

\section{RESULTS}

\section{Clinical Presentation}

The patient (designated CF-00034) was a previously healthy 14 -yr-old male who presented to his pediatrician with a $3 \mathrm{wk}$ history of coughing and shortness of breath during bicycle riding, with no history of fever. On physical examination, temperature, blood pressure, and oxygen saturation were all in normal ranges. He had decreased breath sounds on the left lower lung base and egophony. The right side was clear to auscultation with good aeration throughout. A chest radiograph was obtained (Fig. 1A), which showed a large left pleural effusion causing compression of the left lung. The patient then underwent a video-assisted thoracoscopic procedure to drain the effusion. A thick, straw-colored fluid was removed, and multiple pleural-based and hilar masses were noted and biopsied (Fig. 1B-D) before the lung was reexpanded. The biopsies showed sheets of small round blue cells with increased mitotic activity that stained positive for myosin, desmin, CD56, and WT-1. Staining for myogenin was also performed and was positive in $80 \%$ of the patient's cells. Fluorescence in situ hybridization (FISH) testing revealed a PAX3-FOXO1 fusion, conferring a final diagnosis of alveolar rhabdomyosarcoma. Staging evaluation revealed a primary mass in the upper left abdomen near the left adrenal gland, along with metastatic disease in both 
A

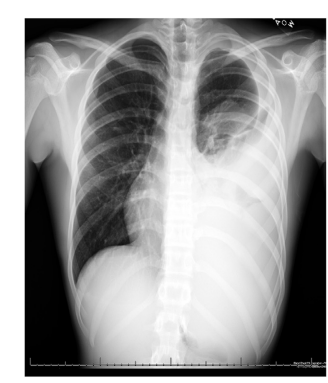

B

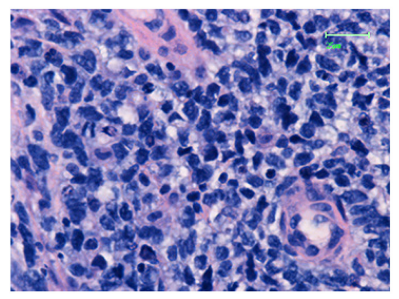

E

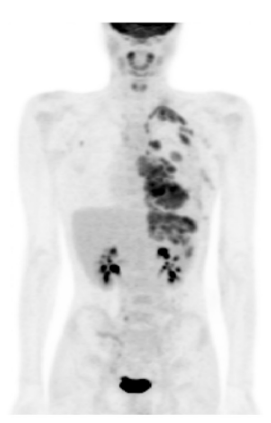

C

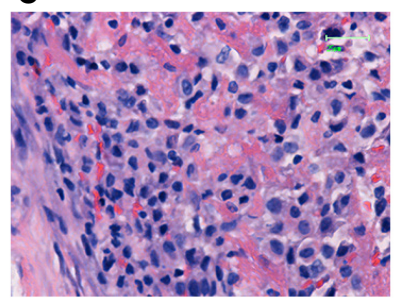

$\mathbf{F}$

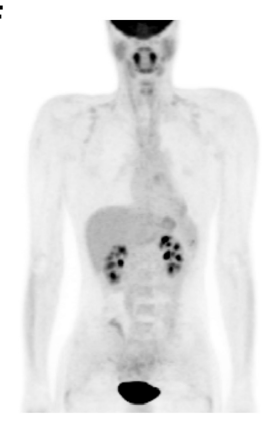

D

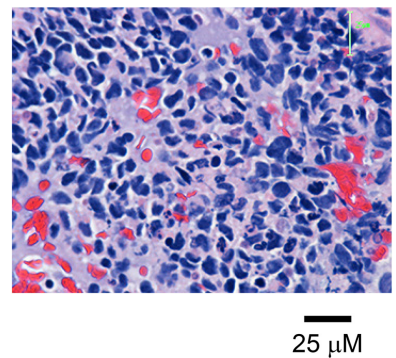

Figure 1. Patient CF-00034 chest scans and biopsy histopathology. (A) Patient initial chest X-ray. (B-D) Representative histology images of hematoxylin and eosin-stained tumor biopsy tissue (dark blue, cell nuclei; pink, extracellular matrix). (E) Patient initial positron emission tomography (PET) scan at diagnosis. (F) Patient PET scan at conclusion of primary therapy.

lungs and hilar and supraclavicular lymphadenopathy. Bone marrow was not involved. On further questioning, the mother also reported that multiple family members on the maternal side had been diagnosed with malignant hypertension, pheochromocytomas, and thyroid cancer. Germline genetic testing on the patient revealed a c.1091G > T (p.C634F) mutation in the RET gene, consistent with a diagnosis of multiple endocrine neoplasia type 2A (MEN2A). Multiple family members, including the patient's mother, underwent genetic testing as well, and the same RET mutation was identified. This particular mutation is associated with a high risk of medullary thyroid cancer, and current clinical guidelines recommend early biochemical screening and prophylactic thyroidectomy at or before the age of 5 (Spinelli et al. 2016). Patients with mutant RET also have an increased risk of pheochromocytoma and hyperparathyroidism.

The patient was started on chemotherapy with irinotecan, vincristine, cyclophosphamide, doxorubicin, dactinomycin, ifosfamide, and etoposide. He had good initial response to therapy, with resolution of his malignant effusion and lung lesions after $2 \mathrm{mo}$. He underwent radiation to the primary abdominal lesion as well as whole-lung radiation, before proceeding with more chemotherapy. Compared to the PET scan at diagnosis showing disease in the lung and abdomen (Fig. 1E), at the end of first-line therapy, the patient had resolution of fluorodeoxyglucose (FDG) uptake and no evidence of disease by imaging (Fig. 1F). The patient was then started on a cabozantinib (a RET inhibitor) in the context of "off-label" usage given that cabozantinib is an FDA-approved agent for the treatment of cancer, but not rhabdomyosarcoma specifically.

No evidence in the literature yet existed that a RET inhibitor would be efficacious in preventing or delaying relapse of a germline RET-mutant alveolar rhabdomyosarcoma, but given the poor prognosis of metastatic alveolar rhabdomyosarcoma and the mutant RET status, the patient and family elected to use a commercially available RET inhibitor (RETi) off label as 
maintenance therapy for a planned duration of a year. Standard adult dosing was used given the patient's size and lack of pediatric data at the time. While on maintenance therapy, he underwent imaging every 3 mo. After 6 mo of therapy, the patient took himself off the medication. He had no evidence of disease on imaging at that time. However, he developed new lung nodules on surveillance imaging 3 mo later, and biopsy confirmed recurrent rhabdomyosarcoma.

Cessation of the RET inhibitor may not have had any effect on the timing of relapse, but the family noted that while cabozantinib was being taken the drug was generally well-tolerated. The patient did have some mild grade 1 thyroid dysfunction, which is commonly reported with these inhibitors. Screening biochemical studies including parathyroid, calcium, phosphorus, calcitonin, and catecholamine levels in this patient were all normal and remained normal throughout his treatment. No multiple endocrine neoplasia (MEN)related tumors were ever seen on imaging.

\section{Genomic Analyses}

Tumor tissue resected from an early operation was stored as formalin-fixed, paraffin-embedded tissues, which were sectioned and sent for DNA isolation and whole-exome sequencing through our CLIA-certified sequencing partner Beijing Genomics Institute (the complete process sequencing and analysis process was not CLIA-certified and would be classified as research-level). White blood cells isolated from a blood draw were sent for DNA isolation and whole-exome sequencing as matched normal tissue. Tissue resected during a later operation following recurrence was sent for DNA and RNA isolation and sequencing. The total sequencing data set thus consisted of whole-exome sequencing of the initial tumor, wholeexome sequencing of matched normal DNA from blood, whole-exome sequencing of relapsed tumor tissue, and whole-transcriptome sequencing of relapse tumor tissue. This sequencing data set was analyzed for both pathogenic germline alterations and actionable (druggable) targets based on the presence of somatic missense mutations altering the function of the original gene, evidence of copy-number gain, and level of gene expression. A circos plot of genomic alterations, at the time of relapse, is provided in Figure 2A, and an abbreviated set of sequencing results is provided in Figure 2B. A table of relevant genomic variations is provided in Table 1. Coverage for sequencing experiments is provided in Table 2. Larger version circos plots documenting genomic alterations of initial and relapse tumors can be found in Supplemental Figure 1, a partial list of sequencing results can be found in Supplemental Table 1, and complete sequencing results are presented in Supplemental Material.

\section{RET C634F}

Initial attempts to generate patient-derived primary cultures were unsuccessful, which necessitated identifying cell models biosimilar to the index case for in vitro experiments to validate potential therapy options. Similarity of cell models was quantified using an ARMS-focused dendrogram computational model. This analysis is being prepared for a separate manuscript (KA Crawford, NE Berlow, C Keller, in prep.) focusing on RET activation in the broader context of ARMS. When biopsy material became available from relapse surgeries, western blot analysis of CF-00034 revealed the absence of RET protein (Fig. 3), which is consistent with low expression of RET per RNA sequence analysis (0.05 TPM; Fig. 2B).

Throughout this study the evaluation of patient-specific therapeutic strategies was hindered by the lack of a patient-derived cell model. To overcome this limitation, the patient engaged the services of Champions Oncology for the generation of a mouse patientderived xenograft (PDX) model (CTG-3483). Tumors explanted from the PDX mice were developed into a primary cell culture that was used for drug screen studies. The resulting 
COLD SPRING HARBOR Molecular Case Studies
Genetic analysis and drug intervention in ARMS

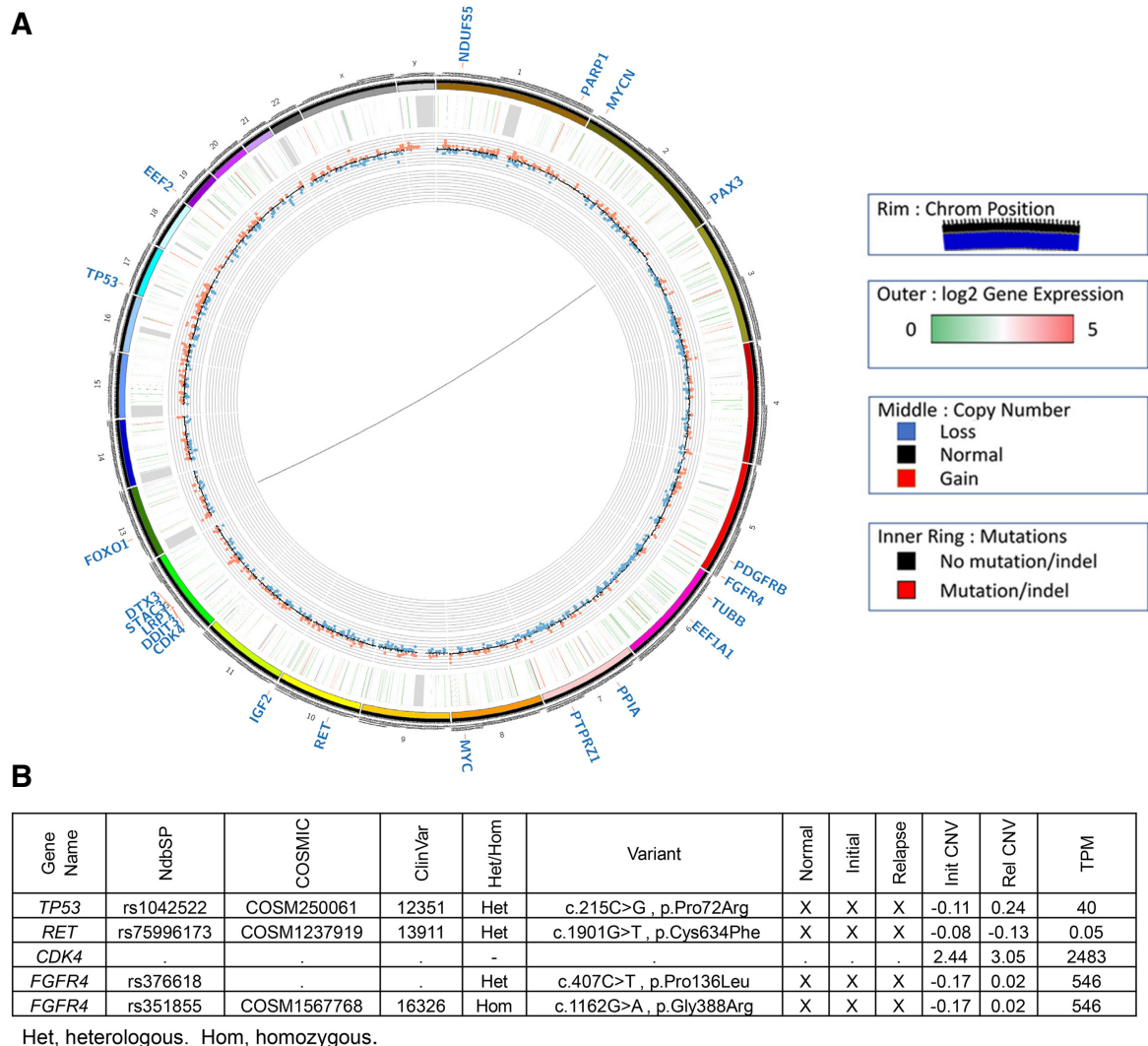

Figure 2. Circos plot of patient's molecular sequencing. (A) Genetic alterations of relapse biopsy. Initial biopsy results provided in Supplemental Figure 1. (B) DNA whole-exome and RNA whole-transcriptome sequencing results of high interest. (C) $\mathrm{EC}_{50}$ values for cell models treated with single-agent Stattic.

culture (CF-00034-5) was confirmed for PAX3-FOXO1 fusion by western blot and authenticated through short tandem repeat (STR) analysis (Supplemental Fig. 2). CF-00034-5 was used to evaluate the effectiveness of RETi cabozantinib, the drug used for maintenance therapy. The results (Fig. 3B,C) indicate that CF-00034-5 is only sensitive to cabozantinib (EC 50 $11 \mu \mathrm{M}$ ) at concentrations above that which can be achieved clinically (100 mg daily dose for 2 wk resulted in a mean plasma concentration of $2.2 \mu \mathrm{M}$ ) (Daud et al. 2017).

Nonetheless, mutations in RET are known to lead to constitutive activity of RET signaling (Pecce et al. 2018), which might present a strong target for RET inhibition. However, the lack of sensitivity to multiple RET inhibitors and the absence of RET protein expression suggest the patient's germline RET mutation was not biologically relevant in this rhabdomyosarcoma. Newly available clinical RET inhibitors have shown promising results in typically RET-associated cancers, such as BLU 667 in RET-fusion positive NSCLC (Gainor et al. 2019) and LOXO-292 in RET mutant cancers such as lung cancer and thyroid cancer (Guo et al. 2019). These nextgeneration RET inhibitors may prove more effective than previously validated inhibitors.

\section{CDK4 Amplification}

The 12q13-q14 amplicon is common in RMS, found in 25\% of PAX3-FOXO1 tumors and $4 \%$ of PAX7-FOXO1 tumors (Olanich et al. 2015). This amplification is associated with an aggressive ARMS subtype and contains cyclin-dependent kinase 4 (CDK4) locus. CDK4/6 inhibitors have been approved for use in metastatic breast cancer and are being investigated 


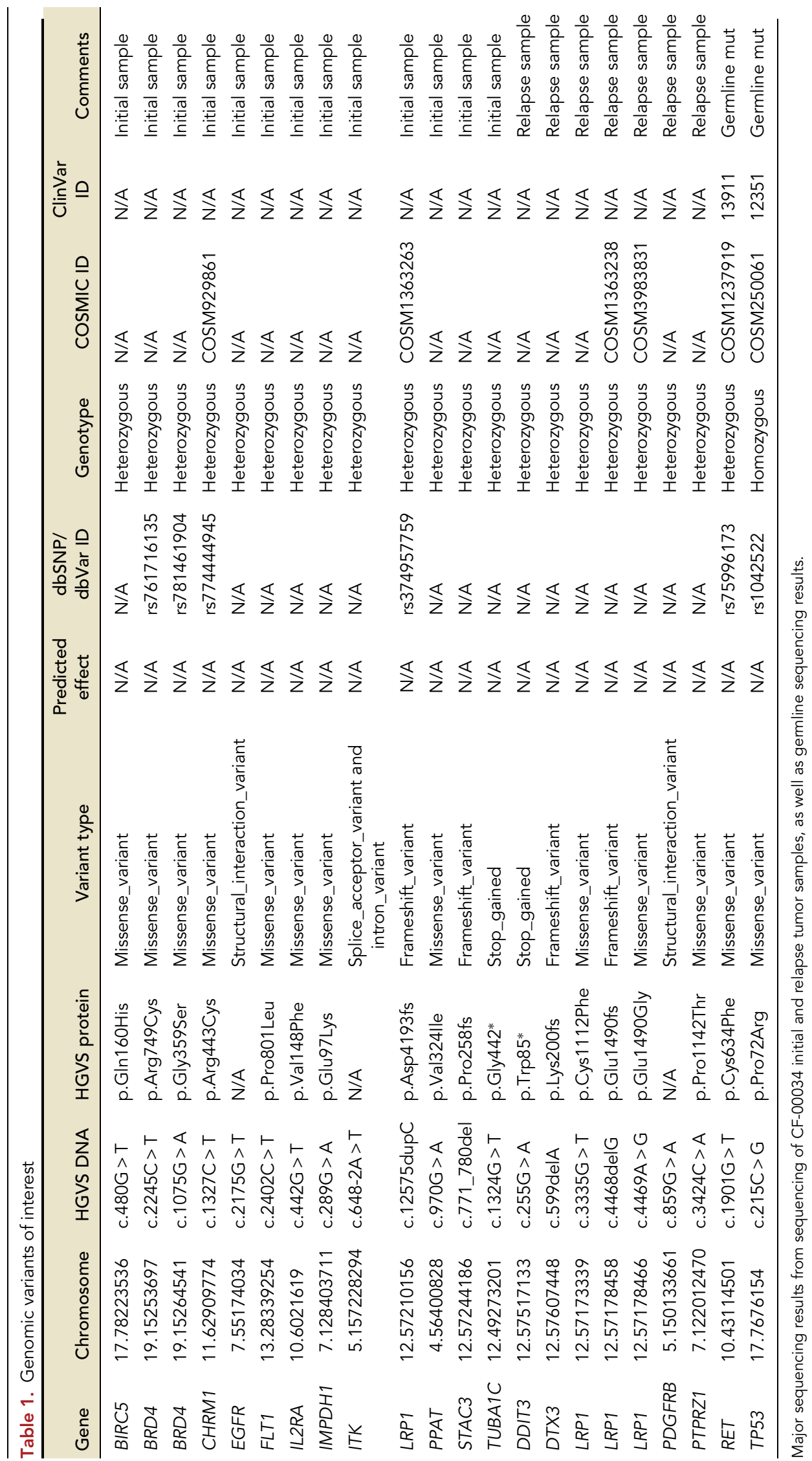


Table 2. Sequencing experiment coverage

\begin{tabular}{llcl}
\hline Sample name & \multicolumn{1}{c}{ Sequencing type } & Sample type & \multicolumn{1}{c}{ Coverage } \\
\hline CF-00034 initial tumor & DNA whole exome & Tumor & $100 \times$ (paired end) \\
CF-00034 relapse tumor & DNA whole exome & Tumor & $100 \times$ (paired end) \\
CF-00034 normal sample & DNA whole exome & Normal & $50 \times$ (paired end) \\
CF-00034 RNA tumor & RNA whole transcriptome & Tumor & $40 \mathrm{M}$ reads (paired end) \\
\hline
\end{tabular}

Sequencing type, sample type, and coverage for sequencing experiments performed in this manuscript.

for a multitude of other solid tumors as well as sarcomas, including RMS (Schettini et al. 2018). Although CDK4 inhibition has been found to be effective in CDK4-amplified liposarcoma and neuroblastoma, CDK inhibitor use in rhabdomyosarcoma is not supported by recent findings (Olanich et al. 2015). To this point, the effectiveness of LEE011 (ribociclib, a specific CDK4/CDK6 inhibitor) in rhabdomyosarcoma correlated across cell lines with low expression of $C D K 4$, with results contrary to the intuitive hypothesis. Based on these results, we chose not to pursue CDK4/6 inhibitor studies.

\section{FGFR4 G388R P136L}

FGFR4 G388R is a common polymorphism found in $50 \%$ of the human population. This polymorphism (ClinVar16326) is associated with cancers of bone, breast, colon, prostate, skin, lung, head and neck, soft-tissue sarcoma, and non-Hodgkin's lymphoma (Frullanti et al. 2011). The Arg388 residue is located in the cytoplasmic juxtamembrane region and is suggested to expose a novel STAT3 binding site (Ulaganathan et al. 2015). As such, we evaluated ARMS cell line Rh30 and embryonal RMS cell line Rh6 sensitivities to pathway inhibitors including FGF401 (FGFR4), PP1 (SRC), ruxolitinib (JAK1/2), and Stattic (STAT3). Both Rh30 and Rh6 are isogenic to CF-00034 with respect to FGFR4 G388R P136L status. Of all of these agents, only Stattic demonstrated consistent proliferation inhibition, albeit with half-maximal effective concentration ( $50 \%$ cell viability dosage point, $\mathrm{EC}_{50}$ ) $>2 \mu \mathrm{M}$ (Fig. 4). Stattic is a nonpeptidic small molecule and was chosen for this study because of its reported selectivity for STAT3 over STAT1/5/6 inhibition (IC 50 of $5.1 \mu \mathrm{M}$ [Schust et al. 2006]). However, more recent studies have determined that Stattic is a reactive compound

A

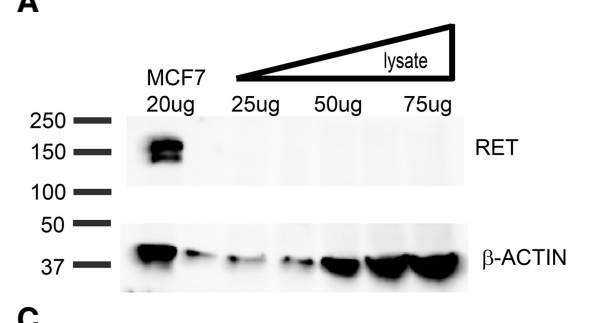

B

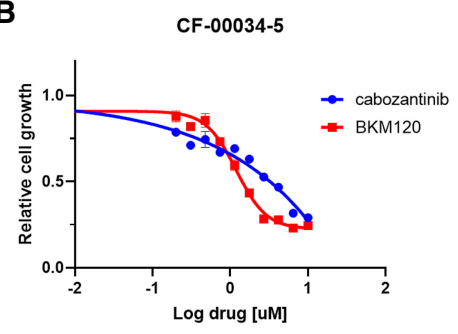

C|c|c|
\begin{tabular}{|c|c|c|}
\hline & cabozantinib $(\mu \mathrm{M})$ & BKM120 $(\mu \mathrm{M})$ \\
\hline $\mathrm{C}_{\max }$ & 4 & 3 \\
\hline $\mathrm{EC}_{50}$ CF-00034-5 & 11 & 1.3 \\
\hline
\end{tabular}

Figure 3. CF-00034 biopsy RET expression and CF-00034-5 cell culture sensitivity to RETi. (A) Western blot analysis of CF-00034 tumor biopsy at time of relapse. The MCF7 breast cancer cell line is known to express RET and was used as a positive control. (B) Cell proliferation curves of CF-00034 derived cell culture. Cabozantinib is a highly selective RETi that the patient received as maintenance therapy, and BKM120 (PI3Ki) was used as a positive control. (C) Table of reported drug $C_{\max }$ values and $\mathrm{EC}_{50}$ values determined for CF-00034-5. 
A

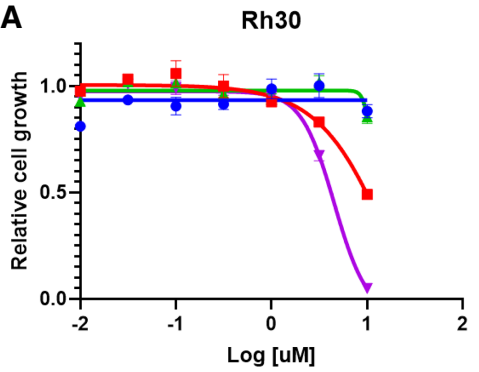

B

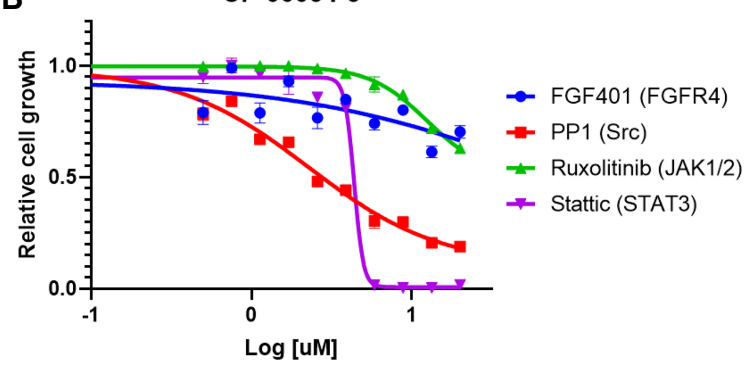

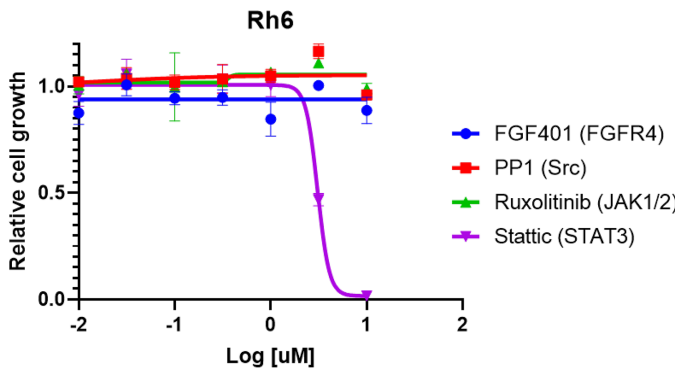

C

\begin{tabular}{|c|c|}
\hline & stattic EC $_{50}$ \\
\hline Rh30 & $3.7 \mu \mathrm{M}$ \\
\hline Rh6 & $2.9 \mu \mathrm{M}$ \\
\hline CF-34-5 & $4.4 \mu \mathrm{M}$ \\
\hline
\end{tabular}

Figure 4. Proliferation inhibition of RMS FGFR4 G388R cell lines. (A) Cell proliferation curves for Rh30 (ARMS) and Rh6 (ERMS) with JAK/STAT pathway inhibitors. (B) STAT3 inhibitor Stattic inhibited cellular proliferation of RMS cell lines Rh30 and Rh6 that bearing FGFR4 G388R P136L. (C) EC 50 values for cell models treated with single-agent Stattic.

that alkylates cysteines indiscriminately, and is thus a potentially promiscuous probe for evaluating STAT3 biology.

\section{Functional Analyses; Drug Screening of Patient-Derived Cell Culture}

The results of a 60-compound drug screen are shown in Table 3. The drug screen is composed of approved drugs, drugs in late stage development, and drugs enriched in inhibitors of epigenetic targets. Two of the most potent drugs identified from the screen were the PI3K inhibitor BKM120 and HSP90 inhibitor CUDC-305, which had no correlative genomic alterations for this patient. We extended this study further by performing drug synergy studies with PI3K inhibitors BKM120 and BYL719, and HSP90 inhibitor AUY922 (Fig. 5A). These studies demonstrate that BKM120 was a more effective inhibitor of proliferation than BYL719 (Fig. 5B), and both drugs demonstrated synergy with HSP9O inhibitor AUY922 at clinically achievable concentrations (Fig. 5C,D).

Although the screen did not contain a highly specific RET inhibitor, two RTK inhibitors (dasatinib and midostaurin) are moderately selective inhibitors of RET; despite both drugs being tested at more than 10 times the agent's RET $K_{d}$ concentration, neither drug demonstrated single agent activity (Supplemental Table 2). Similarly, the potent pan-FGFR inhibitor LY2874455 showed minimal in vitro efficacy, despite the presence of two FGFR4 mutations (Supplemental Table 2).

\section{DISCUSSION}

We performed extensive genomic analysis of a 14-yr-old patient (carrying a germline RETactivating mutation) diagnosed with metastatic rhabdomyosarcoma with the interest of 


\begin{tabular}{|c|c|c|}
\hline Drugs & Target & Absolute EC50 (nM) \\
\hline Dinaciclib ${ }^{a}$ & CDK 2/5/1/9 & 10 \\
\hline INK128 & mTORC1,2 & 70 \\
\hline Panobinostat ${ }^{a}$ & pan-HDAC & 150 \\
\hline Brefeldin $A^{a}$ & ATPase & 150 \\
\hline Thapsigargin & SERCA & 630 \\
\hline Mithramycin A & SP1 & 770 \\
\hline BKM120 & PI3K $(p 110 a, \delta, \beta, \gamma)$, mTOR & 990 \\
\hline CUDC-305 & HSP9O & 1070 \\
\hline YM155 & Survivin & 1200 \\
\hline BIX 01294 & G9a (H3K9me2) & 1430 \\
\hline BMS-754807 & IGF1R, AURKA/B & 1710 \\
\hline UNC0642 & G9a/GLP & 1730 \\
\hline SGI-1776 & $\mathrm{PIM} 1 / 2 / 3$ & 1850 \\
\hline Dasatinib & Abl, Src, and c-Kit & 4840 \\
\hline Crizotinib & C-MET, ALK & 5240 \\
\hline CUDC907 & $\mathrm{HDAC} 1 / 2 / 3 / 6 / 10 / 11, \mathrm{PI} 3 \mathrm{Ka}$ & 6700 \\
\hline
\end{tabular}

Top hits from 60 compound drug candidate screen used for target identification for CF-00034. ${ }^{a}$ Frequent inhibitors of proliferation in vitro.

creating generalized knowledge for the literature that might address the use of RET inhibitors for rhabdomyosarcoma. Analysis of the patient's initial and relapse tumor tissue confirmed the patient's RET germline mutation and identified a small set of potentially actionable variations, which were evaluated in vitro once a primary cell culture became available. Despite the presence of RET C634F mutation, RET itself was minimally expressed at the transcript level and showed no evidence of protein expression by western blot, suggesting that RET inhibitor therapy might have been unlikely to be effective frontline therapy for this patient. This observation was confirmed once the patient-derived cell culture was developed and assayed for sensitivity to RET inhibitor cabozantinib. The cell culture was sensitive to cabozantinib but only at concentrations above what is achievable in the clinic. The modest sensitivity to cabozantinib can be explained by the drug's inhibition of homologous tyrosine kinases such as KIT, KDR, $A X L$, and $\mathrm{MET}\left(K_{d}=4.6,7,7.5\right.$, and $9 \mathrm{nM}$, respectively) (Tanoli et al. 2018), which bind to these targets at lower concentrations than RET (18 nM) or off-target effects at micromolar concentrations. Additional results were obtained from the 60-compound drug screen. Although the screen did not contain a highly specific RET inhibitor, the screen included nine tyrosine kinase inhibitors including two RTK inhibitors (dasatinib and midostaurin) that are moderately selective inhibitors of RET; despite both drugs being tested at 10 times the agent's RET $K_{d}$ concentration, neither drug demonstrated single-agent activity. Although the data does not support the use of RETi as a frontline therapy for this patient, the value of using a multikinase inhibitor, such as cabozantinib, for maintenance therapy remains an open question.

The CF-00034 patient was found to have the 12q13-q14 amplicon that contains the CDK4 locus and to have high CDK4 transcription. However, contrary to other cancer types, in fusion-positive RMS, CDK4 amplification does not correlate with in vitro sensitivity to CDK4 inhibitors (Olanich et al. 2015). Consequently, sensitivity to CDK4 inhibitors was not evaluated in this report. We noted, however, that the patient was prescribed palbociclib (CDK4/6i) to treat relapse disease. 
A

\begin{tabular}{|c|c|c|}
\hline drug & target & C $_{\max }$ \\
\hline BKM120 & PI3K $\alpha$ & $3 \mu \mathrm{M}$ \\
\hline BYL719 & PI3K $\alpha$ & $6-11 \mu \mathrm{M}$ \\
\hline AUY922 & HSP90 & $2 \mu \mathrm{M}$ \\
\hline
\end{tabular}

B

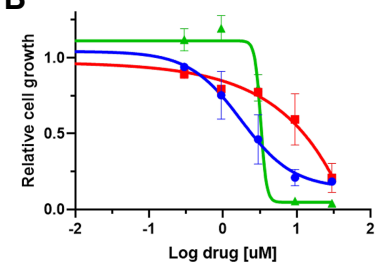

D

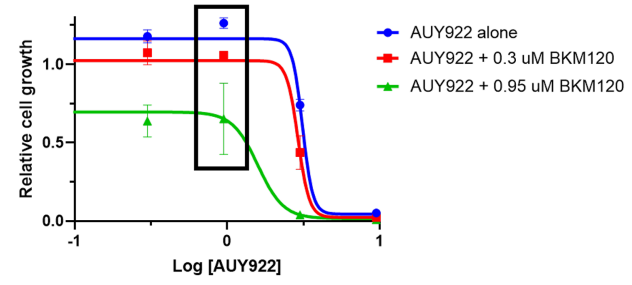

C
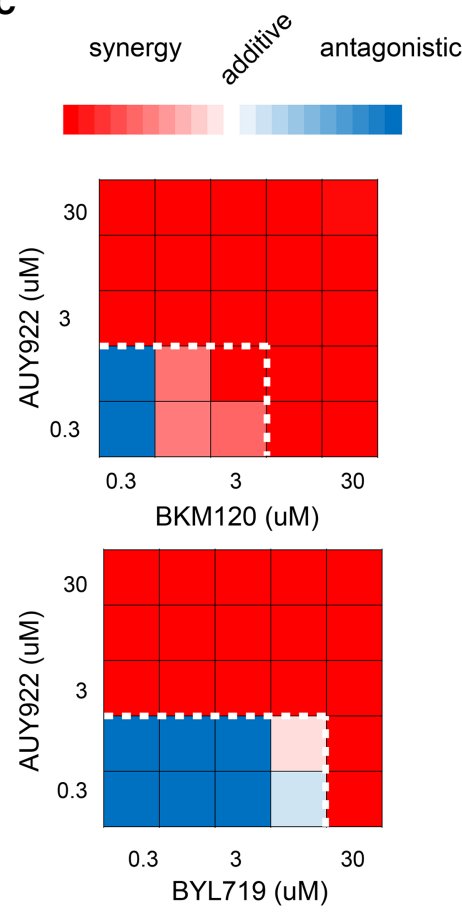

Figure 5. Validation of PI3K/HSP90 as drug targets. (A) Table of PK data of pharmaceuticals used in study. (B) Determination of single-agent $E_{50}$ values. (C) Heatmap of combination indexes calculated at various drug exposures. Dashed lines indicate clinically achievable drug concentrations. (D) Single-agent and drug combination dose-response curves at clinically achievable concentrations (boxed).

The patient's tumor is homozygous for FGFR4 G388R and heterozygous for FGFR4 P136L, and FGFR4 overexpression was observed. The prevalence of the FGFR G388R allowed us to investigate JAK-STAT pathway inhibitors in FGFR G388R mutant RMS cell lines (Rh30 and Rh6), which demonstrated that Stattic (a STAT3 inhibitor) inhibited cellular proliferation. This result was later confirmed with the patient-derived cell culture. Stattic has been reported to block STAT3 dimerization and, thus, nuclear translocation. Several more recent reports have expanded the mechanistic understanding and suggest that Stattic alkylates the cysteines of STAT3 (Heidelberger et al. 2013) and is a nonselective cysteine alkylating agent (Ball et al. 2016). Thus, Stattic is a nonideal probe for interrogating STAT3-related biology. At this point, we have not yet validated any targets in the JAK-STAT pathway for RMS patients by other means.

Functional profiling of the patient's xenograft-derived cell culture revealed sensitivity to both PI3K inhibition and HSP90 inhibition. To further evaluate these oncogenic targets, we validated the PI3K inhibitors BYL719 (alpelisib) and BKM120 (buparlisib) in combination with HSP90 inhibitor AUY922 (luminespib). Each drug demonstrated single agent activity with $\mathrm{EC}_{50}$ values near their reported clinically achievable concentrations, and both PI3K inhibitors showed in vitro synergy with AUY922 for this patient's cell model. Evaluating BKM120AUY922 combinations in RMS is open for further evaluation.

Though rare in the population and especially within the context of ARMS, the RET C634F germline mutation presents a challenging clinical phenotype. While associated with the development of cancers, RET C634F may be minimally involved in tumor progression and maintenance and likely did not confer sensitivity to RET inhibition. Thus, RET inhibition may not be an effective therapeutic option once disease is progressing. Nonetheless, the 
COLD SPRING HARBOR Molecular Case Studies
Genetic analysis and drug intervention in ARMS patient's course of treatment cannot exclude the possibility that RET inhibitor therapy may have impacted time to recurrence and might be studied in the context of a maintenance therapy for RET-mutant ARMS when disease is in remission.

For this patient, high-throughput exome and transcriptome sequencing provided actionable results, but based on functional testing, these results may have provided little clinical benefit. The RET C634F mutation did not confer RET sensitivity, CDK4 amplification is inversely related with CDK4 inhibitor sensitivity in RMS, and the FGFR4 mutations did not directly induce FGFR sensitivity, though did reveal a potential sensitivity to STAT3 inhibitors. Unfortunately, potent STAT3 inhibitors are not yet available for clinical use, but are worth exploration when clinically relevant inhibitors become available. Instead, functional testing pointed to targets that would not likely have been selected from high-throughput sequencing-specifically, PI3K inhibitors and HSP9O inhibitors. Thus, for this patient, high-throughput sequencing would likely have less ideally informed effective clinical treatment options based on functional testing results. When available, functional testing may provide a valuable supplement to, or even a primary methodology for, therapy assignment for high-risk cancer such as ARMS.

\section{METHODS}

\section{Cell Lines}

The Rh30 cell line (Douglass et al. 1987) (RRID:CVCL_0041) was shared by the Children's Oncology Group Childhood Cancer Repository and was cultured in RPMI-1640 (11875-093; ThermoFisher Scientific) supplemented with 10\% fetal bovine serum (FBS) (26140079; ThermoFisher Scientific) and 1\% penicillin-streptomycin (15140-122; ThermoFisher Scientific) (RPMI+10:1) in $5 \% \mathrm{CO}_{2}$ at $37^{\circ} \mathrm{C}$, per cell line instructions. Rh6 and Rh3 cell lines were obtained from Dr. P. Houghton of St. Jude Children's Research Hospital, Memphis, TN, and was cultured in RPMI+10:1. MCF-7 (ATCC HTB-22) was obtained from ATCC and cultured in ATCC-formulated Eagle's Minimum Essential Medium (30-2003, ATCC) similarly supplemented with FBS and penicillin-streptomycin.

\section{Western Blot}

Soluble protein lysates were prepared using RIPA buffer (Thermo 89900), with HALT protease and phosphatase inhibitor cocktail (Thermo 78441). An amount of $20 \mu \mathrm{g}$ of MCF7 protein lysate was run on 10\% Tris-Glycine SDS-polyacrylamide gel (Bio-Rad 456-1024). CF-00034 tumorbiopsy material was washed with cold PBS, minced by hand, and further processed in RIPA + HALT using an immersion sawtooth generator probe, and sonication. CF-00034 soluble protein lysate was loaded in a range of concentrations. After electrophoresis, the protein gel was transferred to PVDF membrane (Bio-Rad 1620177). For Figure 3, membranes were incubated with primary antibodies: RET (Cell Signaling 3223) and $\beta$-actin (Sigma-Aldrich A5441). After appropriate washing, HRP-conjugated secondary antibody (anti-rabbit PI-1000, and antimouse PI-2000, Vector Laboratories) incubation was performed. Blots were visualized with chemiluminescent substrates (Bio-Rad 1705061). For Supplemental Figure 3 western blot, primary antibodies used consist of PAX3 antibody (R\&D Systems MAB2457) and GAPDH (Cell Signaling Technology 2118) and the same secondary antibodies as mentioned above.

\section{Cellular Proliferation Assay}

Rhabdomyosarcoma cell lines Rh30 and Rh6 were seeded in 384-well plates at 2500 cells per well, in $30 \mu \mathrm{L}$ of media, using a BioTek MultiFlo dispenser. Cultures were grown overnight and then treated with drugs FGF401 (23029), PP1 (14244), Ruxolitinib (11609), and Stattic 
COLD SPRING HARBOR Molecular Case Studies
Genetic analysis and drug intervention in ARMS

(14590) that were all purchased from Cayman Chemical. All of the pharmaceuticals were administered at the following concentrations using a D300e digital dispenser (Tecan Trading AG): $0.01,0.0316,0.1,0.316,1,3.16$, and $10 \mu \mathrm{M}$. After $72 \mathrm{~h}$ of drug treatment, cultures were treated with an equal volume of CellTiter-Glo reagent (Promega 2020-01-29). Luminescence was measured in a Biotek Instruments Inc. plate reader. The data was then analyzed using Prism GraphPad software.

\section{Primary Cell Culture Generation}

Tumor tissue resected from the patient was sent to Champions Oncology for development of an avatar mouse model (denoted CTG-3483) using their previously published methodology (Rubio-Viqueira et al. 2006; Hidalgo et al. 2011). Once the patient CF-00034 avatar model was established, a PDX tumor explant were received from Champions Oncology model CTG-3483 (study 1130-009, animal ID 120492 and 129494). Tumor tissue was minced by hand and processed with GentleMacs dissociator (130-093-235, Miltenyi Biotec GmbH) according to the manufacturer's protocol. The cultures were grown in RPMI + 10:1. The resulting primary cell culture was designated CF-00034-5.

All animal procedures performed at Champions Oncology were conducted in accordance with the Guidelines for the Care and Use of Laboratory Animals and were approved by the Institutional Animal Care and Use Committee at Champions Oncology.

\section{Novel Drug Screen}

A set of 60 drug candidates were applied to 384-well plates, in triplicate, to generate a four-point dose curve (final concentrations of $0.01,0.1,1$, and $10 \mu \mathrm{M}$ ). Primary cell culture CF-00034-5 was added to the plates using MultiFlo (BioTek) dispenser, and plates were incubated for $72 \mathrm{~h}$. Cellular proliferation was then measured using Cell Titer Glo as mentioned above. $I_{50}$ values were determined using Excel (Microsoft) software.

\section{Drug Combination Studies}

Primary cell line CF-00034-5 was prepared in a 384-well plate for cellular proliferation assay in an identical manner as above. After overnight incubation, the cultures were treated with inhibitors BKM120 (HY-70063, MedChemExpress LLC), BYL719 (S2814, Selleck Chemical), and AUY922 (HY-10215, MedChemExpress). All of the pharmaceuticals were administered at final concentrations of $0.3,0.949,3,9.49$, and $30 \mu \mathrm{M}$. Cellular proliferation was measured by Cell Titer Glo as described above. IC 50 values were determined using GraphPad software and combination indexes were calculated using CalcuSyn software (Biosoft).

\section{ADDITIONAL INFORMATION}

\section{Data Deposition and Access}

All sequencing data, both DNA whole-exome and RNA whole-transcriptome, are available through the European Genome-phenome Archive (EGA; https://ega.archive .org), study accession ID EGAS00001004359. Sequencing data for this project is protected and can be requested through the EGA system under the data access policies established for the study.

\section{Ethics Statement}

All human tissue samples were acquired through the Cancer Registry for Familial and Sporadic Tumors (CuRe-FAST) tumor banking program. All patients enrolled in CuReFAST provided informed consent. All aspects of the study were reviewed and approved 
Competing Interest Statement The authors have declared no competing interest.

Referees

Vivek Subbiah

Anonymous

Received October 15, 2019; accepted in revised form April 13, 2020. by the Children's Cancer Therapy Development Institute (cc-TDI) Institutional Review Board (IRB). Patient data and clinical and pathologic information are maintained in a de-identified database.

\section{Acknowledgments}

This work was funded by the Genomic Endotypes in Alveolar Rhabdomyosarcoma (GEAR) project consortium on Consano.org. STR profiling was performed by the University of Arizona Genetics Core, University of Arizona, Tucson, AZ..

\section{Author Contributions}

K.A.C. and N.E.B. designed experiments, interpreted results, and wrote the manuscript; J.T. and M.L. wrote the manuscript; T.H. treated the patient and wrote the manuscript; M.M. and C.N. generated the mouse PDX model; and C.K. designed the experiments and wrote the manuscript.

\section{REFERENCES}

Ball DP, Lewis AM, Williams D, Resetca D, Wilson DJ, Gunning PT. 2016. Signal transducer and activator of transcription 3 (STAT3) inhibitor, S3l-201, acts as a potent and non-selective alkylating agent. Oncotarget 7: 20669-20679. doi:10.18632/oncotarget.7838

Daud A, Kluger HM, Kurzrock R, Schimmoller F, Weitzman AL, Samuel TA, Moussa AH, Gordon MS, Shapiro GI. 2017. Phase II randomised discontinuation trial of the MET/NEGF receptor inhibitor cabozantinib in metastatic melanoma. Br J Cancer 116: 432-440. doi:10.1038/bjc.2016.419

Douglass EC, Valentine M, Etcubanas E, Parham D, Webber BL, Houghton PJ, Houghton JA, Green AA. 1987. A specific chromosomal abnormality in rhabdomyosarcoma. Cytogenet Cell Genet 45: 148-155. doi:10 $.1159 / 000132446$

Frullanti E, Berking C, Harbeck N, Jezequel P, Haugen A, Mawrin C, Parise O Jr, Sasaki H, Tsuchiya N, Dragani TA. 2011. Meta and pooled analyses of FGFR4 Gly388Arg polymorphism as a cancer prognostic factor. Eur J Cancer Prev 20: 340-347. doi:10.1097/CEJ.0b013e3283457274

Gainor JF, Lee DH, Curigliano G, Doebele RC, Kim D-W, Baik CS, Tan DS-W, Lopes G, Gadgeel SM, Cassier PA, et al. 2019. Clinical activity and tolerability of BLU-667, a highly potent and selective RET inhibitor, in patients (pts) with advanced RET-fusion+ non-small cell lung cancer (NSCLC). J Clin Oncol 37: 9008. doi:10 .1200/JCO.2019.37.15_suppl.9008

Guo R, Schreyer M, Chang JC, Rothenberg SM, Henry D, Cotzia P, Kris MG, Rekhtman N, Young RJ, Hyman $D M$, et al. 2019. Response to selective RET inhibition with LOXO-292 in a patient with RET fusion-positive lung cancer with leptomeningeal metastases. JCO Precision Oncol 3: 1-6. doi:10.1200/PO.19.00021

Hawkins DS, Spunt SL, Skapek SX. 2013. Children's Oncology Group's 2013 blueprint for research: soft tissue sarcomas. Pediatr Blood cancer 60: 1001-1008. doi:10.1002/pbc.24435

Heidelberger S, Zinzalla G, Antonow D, Essex S, Basu BP, Palmer J, Husby J, Jackson PJ, Rahman KM, Wilderspin AF, et al. 2013. Investigation of the protein alkylation sites of the STAT3:sTAT3 inhibitor Stattic by mass spectrometry. Bioorg Med Chem Lett 23: 4719-4722. doi:10.1016/j.bmcl.2013.05.066

Hidalgo M, Bruckheimer E, Rajeshkumar NV, Garrido-Laguna I, De Oliveira E, Rubio-Viqueira B, Strawn S, Wick MJ, Martell J, Sidransky D. 2011. A pilot clinical study of treatment guided by personalized tumorgrafts in patients with advanced cancer. Mol Cancer Ther 10: 1311-1316. doi:10.1158/1535-7163.MCT-11-0233

Oberlin O, Rey A, Lyden E, Bisogno G, Stevens MC, Meyer WH, Carli M, Anderson JR. 2008. Prognostic factors in metastatic rhabdomyosarcomas: results of a pooled analysis from United States and European cooperative groups. J Clin Oncol 26: 2384-2389. doi:10.1200/JCO.2007.14.7207

Olanich ME, Sun W, Hewitt SM, Abdullaev Z, Pack SD, Barr FG. 2015. CDK4 amplification reduces sensitivity to CDK4/6 inhibition in fusion-positive rhabdomyosarcoma. Clin Cancer Res 21: 4947-4959. doi:10.1158/ 1078-0432.CCR-14-2955

Pecce V, Sponziello M, Damante G, Rosignolo F, Durante C, Lamartina L, Grani G, Russo D, di Gioia CR, Filetti $S$, et al. 2018. A synonymous RET substitution enhances the oncogenic effect of an in-cis missense mutation by increasing constitutive splicing efficiency. PLoS Genet 14: e1007678. doi:10.1371/journal.pgen .1007678 
Raney RB, Walterhouse DO, Meza JL, Andrassy RJ, Breneman JC, Crist WM, Maurer HM, Meyer WH, Parham DM, Anderson JR. 2011. Results of the Intergroup Rhabdomyosarcoma Study Group D9602 protocol, using vincristine and dactinomycin with or without cyclophosphamide and radiation therapy, for newly diagnosed patients with low-risk embryonal rhabdomyosarcoma: a report from the Soft Tissue Sarcoma Committee of the Children's Oncology Group. J Clin Oncol 29: 1312-1318. doi:10.1200/JCO.2010.30 .4469

Rubio-Viqueira B, Jimeno A, Cusatis G, Zhang X, lacobuzio-Donahue C, Karikari C, Shi C, Danenberg K, Danenberg PV, Kuramochi $\mathrm{H}$, et al. 2006. An in vivo platform for translational drug development in pancreatic cancer. Clin Cancer Res 12: 4652-4661. doi:10.1158/1078-0432.CCR-06-0113

Rudzinski ER, Anderson JR, Chi YY, Gastier-Foster JM, Astbury C, Barr FG, Skapek SX, Hawkins DS, Weigel BJ, Pappo A, et al. 2017. Histology, fusion status, and outcome in metastatic rhabdomyosarcoma: a report from the Children's Oncology Group. Pediatr Blood Cancer 64. doi:10.1002/pbc.26645

Schettini F, De Santo I, Rea CG, De Placido P, Formisano L, Giuliano M, Arpino G, De Laurentiis M, Puglisi F, De Placido S, et al. 2018. CDK 4/6 inhibitors as single agent in advanced solid tumors. Front Oncol 8: 608. doi:10.3389/fonc. 2018.00608

Schust J, Sperl B, Hollis A, Mayer TU, Berg T. 2006. Stattic: a small-molecule inhibitor of STAT3 activation and dimerization. Chem Biol 13: 1235-1242. doi:10.1016/j.chembiol.2006.09.018

Spinelli C, Strambi S, Rossi L, Elisei R, Massimino M. 2016. Surgical management of medullary thyroid carcinoma in pediatric age. Curr Pediatr Rev 12: 280-285. doi:10.2174/1573396313666161111120936

Tanoli Z, Alam Z, Vähä-Koskela M, Ravikumar B, Malyutina A, Jaiswal A, Tang J, Wennerberg K, Aittokallio T. 2018. Drug Target Commons 2.0: a community platform for systematic analysis of drug-target interaction profiles. Database 2018: 1-13. doi:10.1093/database/bay083

Ulaganathan VK, Sperl B, Rapp UR, Ullrich A. 2015. Germline variant FGFR4 p.G388R exposes a membraneproximal STAT3 binding site. Nature 528: 570-574. doi:10.1038/nature16449 


\section{COLD SPRING HARBOR Molecular Case Studies}

\section{Case report for an adolescent with germline RET mutation and alveolar rhabdomyosarcoma}

Kenneth A. Crawford, Noah E. Berlow, Jennifer Tsay, et al.

Cold Spring Harb Mol Case Stud 2020, 6: a004853

Access the most recent version at doi: $10.1101 /$ mcs.a004853
Supplementary http://molecularcasestudies.cshlp.org/content/suppl/2020/06/11/mcs.a004853.D Material C1

References This article cites 20 articles, 5 of which can be accessed free at: http://molecularcasestudies.cshlp.org/content/6/3/a004853.full.html\#ref-list-1

License This article is distributed under the terms of the Creative Commons Attribution-NonCommercial License, which permits reuse and redistribution, except for commercial purposes, provided that the original author and source are credited.

Email Alerting Receive free email alerts when new articles cite this article - sign up in the box at the Service top right corner of the article or click here. 